INSTITUT NATIONAL DE RECHERCHE EN INFORMATIQUE ET EN AUTOMATIQUE

\title{
Second order analysis for control constrained optimal control problems of semilinear elliptic systems
}

J. Frédéric Bonnans

\section{$\mathbf{N}^{\circ} 3014$}

Octobre 1996

THÈME 4

\section{apport}

derecherche 



\title{
Second order analysis for control constrained optimal control problems of semilinear elliptic systems
}

\author{
J. Frédéric Bonnans* \\ Thème 4 - Simulation et optimisation \\ de systèmes complexes \\ Projet PROMATH \\ Rapport de recherche $\mathrm{n}^{\circ} 3014$ - Octobre 1996 - 23 pages
}

\begin{abstract}
This paper presents a second-order analysis for a simple model optimal control problem of a partial differential equation, namely a well-posed semilinear elliptic system with constraints on the control variable only. The cost to be minimized is a standard quadratic functional. Assuming the feasible set to be polyhedric, we state necessary and sufficient second order optimality conditions, including a characterization of the quadratic growth condition. Assuming that the second order sufficient condition holds, we give a formula for the second order expansion of the value of the problem as well as the directional derivative of the optimal control, when the cost function is perturbed. Then we partially extend these results to the case of vector valued controls when the feasible set is defined by local and smooth convex constraints. When the space dimension $n$ is greater than 3 , the results are based on a two norms approach, involving spaces $L^{2}(\Omega)$ and $L^{s}(\Omega)$, with $s>n / 2$.
\end{abstract}

Key-words: Optimal control, elliptic systems, sensitivity analysis, expansion of solutions, second order optimality conditions, Legendre forms, polyhedricity, two norms approach.

(Résumé : tsvp)

*Email: Frederic.Bonnans@inria.fr.

Unité de recherche INRIA Rocquencourt

Domaine de Voluceau, Rocquencourt, BP 105, 78153 LE CHESNAY Cedex (France)

Téléphone : (33 1) 39635511 - Télécopie : (33 1) 39635330 


\section{Analyse du deuxième ordre pour les problèmes de commande optimale de systèmes elliptiques semilinéaires}

Résumé : Cet article présente une analyse du second ordre pour un problème modèle de commande optimale d'une équation aux dérivées partielles, à savoir une équation semilinéaire elliptique avec contraintes seulement sur la commande. Le coût à minimiser est une fonctionnelle quadratique standard. Supposant l'ensemble réalisable polyédrique, nous énonçons des conditions nécessaires et suffisantes d'optimalité du deuxième ordre, dont une caratérisation de la condition de croissance quadratique. Supposant vérifiée la condition suffisante du deuxième ordre, nous donnons une formule pour le développement au deuxième ordre de la valeur du problème ainsi que pour la dérivée directionnelle de la solution, lorsque le coût est perturbé. Enfin nous étendons partiellement ces résultats au cas de commandes à valeur vectorielle, l'ensemble réalisable étant défini par des contraintes locales, lisses et convexes. Quand la dimension d'état est supérieures à 3, ces résultats sont basés sur l'approche des deux normes associées aux espaces $L^{2}(\Omega)$ and $L^{s}(\Omega)$, avec $s>n / 2$.

Mots-clé : Commande optimale, systèmes elliptiques, analyse de sensibilité, dévelopement de solutions, conditions d'optimalité, formes de Legendre, polyédricité, approche des deux normes. 
AMS subject classifications 49K40, 49K20, 35B30, 35J60, 90C31.

\section{Introduction}

In this paper we discuss the second order theory for optimal control problems of partial differential equations. This study is related with some recent results in the perturbation theory for abstract optimization problems, $[6,8]$. The results of this paper are obtained by refining the ideas of the abstract theory and adapting them to the structure of optimal control problems. We discuss a simple model problem, that is minimization of a standard quadratic cost with a semilinear elliptic state equation, under various constraints on the control.

The basic tools of our analysis are polyhedricity theory for convex sets $[13,20]$ and the theory of Legendre forms [14]. Combining these tools with some recent progress in the theory of second order conditions for optimality $[8,11]$, we present in section 2 a second order theory that is complete in the sense that there is no gap between the necessary and sufficient conditions. More precisely, we obtain a characterization of the quadratic growth condition (i.e., in the vicinity of the solution, the cost function increases at the same rate that the square of distance to the solution). For a space dimension larger than 3 , the sufficient conditions are formulated in terms of two norms for the control space, i.e. $L^{2}(\Omega)$ and $L^{s}(\Omega)$ for some $s>n / 2$. This may be compared to e.g. [9], where more general nonlinearities are considered, but only sufficient conditions for quadratic growth, using the $L^{\infty}(\Omega)$, are given.

In section 3, assuming a weak second order sufficient condition to hold, and the feasible set to be polyhedric, we provide a formula for computing the directional derivative of the optimal control (as well as a second order expansion of the value function) with respect to a perturbation. This is a partial extension of the results of [6] to the two norms setting, and is to be compared with $[16,23]$ where the derivative of solution is computed under stronger second order conditions, whereas our theory of second order necessary conditions garanties the fact that our sufficient condition is minimal. This result, for $n \geq 3$, takes into account the two norms structure of the problem.

We next generalize in section 4 these results to the case of optimal control problems when the local constraints are defined by a finite number of smooth convex functions. In that case, polyhedricity does not hold, but we show that a theory of second order conditions may be conducted by taking into account the curvature of the constraint functions.

\section{An optimal control problem of elliptic type}

Let $\Omega$ be an open and bounded subset of $\mathbb{R}^{n}$, with $C^{2}$ boundary $\partial \Omega$, whose generic element is denoted $\omega$, and $\phi$ be a $C^{2}$ nondecreasing function $\mathbb{R} \rightarrow \mathbb{R}$. We consider the state equation

$$
\left\{\begin{aligned}
-\Delta y+\phi(y) & =u \text { in } \Omega \\
y & =0 \text { on } \partial \Omega
\end{aligned}\right.
$$

$\mathrm{RR} \mathrm{n}^{\circ} 3014$ 
Here $\Delta$ is the Laplacian operator and $u \in L^{s}(\Omega)$ is the control variable, for some $s \in[2,+\infty)$. The function $y$, defined a.e. over $\Omega$, is called the state, and $\phi(y)$ is the function defined a.e. over $\Omega$ by $\phi(y)(\omega)=\phi(y(\omega))$, a.e. $\omega \in \Omega$.

We recall some basic results of functional analysis. Denote the norm of $L^{s}(\Omega)$ by $\|\cdot\|_{s}$, and the scalar product in $L^{2}(\Omega)$ by $\langle\cdot, \cdot\rangle_{2}$. Let $q=\left(q_{1}, \cdots, q_{\ell}\right)$ be a vector with nonnegative integer components. Set

$$
|q|:=q_{1}+\cdots+q_{\ell} ; \quad D^{q} y:=\frac{\partial^{|q|} y}{\partial x_{1}^{q_{1}} \cdots \partial x_{\ell}^{q_{\ell}}} .
$$

The Sobolev space $W^{m, s}(\Omega)$, with $m$ a non negative integer and $s \in[2,+\infty]$, is defined and endowed with a norm as follows:

$$
\begin{gathered}
W^{m, s}(\Omega):=\left\{y \in L^{s}(\Omega) ; D^{q} y \in L^{s}(\Omega) \text { whenever }|q| \leq m\right\} . \\
\|y\|_{m, s}:=\sum_{0 \leq|q| \leq m}\left\|D^{q} y\right\|_{s} .
\end{gathered}
$$

When $s=2$, we denote $W^{m, 2}(\Omega)$ as $H^{m}(\Omega)$, and endow it with the norm

$$
\|y\|_{m, 2}:=\left(\sum_{0 \leq|q| \leq m}\left\|D^{q} y\right\|_{2}^{2}\right)^{1 / 2} .
$$

The space $W^{m, 2}(\Omega)$ (resp. $H^{m}(\Omega)$ ), endowed with the above norm, is a Banach (resp. Hilbert) space. By the Sobolev imbedding theorem [2, 12], we have

$$
W^{m, s}(\Omega) \subset L^{q}(\Omega), \frac{1}{q}=\frac{1}{s}-\frac{m}{n} \text { if } \frac{1}{s}>\frac{m}{n}, \quad W^{m, s}(\Omega) \subset C(\bar{\Omega}) \text { if } \frac{1}{s}<\frac{m}{n},
$$

the latter inclusion being compact. Let $C(\bar{\Omega})$ (resp. $C_{0}(\Omega)$ ) be the set of continuous functions over $\bar{\Omega}$ (resp. the set of continuous functions over $\bar{\Omega}$ that vanish on $\partial \Omega$ ). The set $C(\bar{\Omega}) \cap$ $W^{m, s}(\Omega)$ is dense in $W^{m, s}(\Omega)$. When $m \geq 1$, the trace on $\partial \Omega$ of an element of $W^{m, s}(\Omega)$ may be defined as a continuous extension from $C(\bar{\Omega}) \cap W^{m, s}(\Omega)$ to $W^{m, s}(\Omega)$ of the trace (in the usual sense), with image in $L^{2}(\partial \Omega)$. The set $W_{0}^{m, s}(\Omega)$ is defined as the closure in $W^{m, s}(\Omega)$ of functions of $C^{\infty}(\bar{\Omega})$ that vanish near the boundary.

We know [3] that for any $u \in L^{s}(\Omega),(2.1)$ has a unique solution $y_{u} \in W^{2, s}(\Omega) \cap W_{0}^{1, s}(\Omega)$, and that there exists $c_{1}>0$ not depending on $u$ such that

$$
\|y\|_{2, s} \leq c_{1}\|u\|_{s} .
$$

In order to obtain differentiability of the mapping $u \rightarrow y_{u}$, it is natural to assume that $s>n / 2,(s=2$ if $n \leq 3)$ so that $W^{2, s}(\Omega) \subset C(\bar{\Omega})$ by $(2.2)$. As $\phi$ is twice continuously differentiable, it is easily proved, using the implicit function theorem, that if $s>n / 2$, the 
mapping $u \rightarrow y_{u}$ is of class $C^{2}: L^{s}(\Omega) \rightarrow W^{2, s}(\Omega)$. In particular, the derivative of $y_{u}$ in direction $v \in L^{s}(\Omega)$ is the unique solution $z \in W^{2, s}(\Omega) \cap W_{0}^{1, s}(\Omega)$ of the linearized system

$$
\left\{\begin{aligned}
-\Delta z+\phi^{\prime}(y) z & =v \text { in } \Omega \\
z & =0 \text { on } \partial \Omega
\end{aligned}\right.
$$

Consider the quadratic cost function

$$
J_{\tau}(y, u):=\frac{1}{2} \int_{\Omega}(y(\omega)-\tau(\omega))^{2} d \omega+\frac{N}{2} \int_{\Omega} u(\omega)^{2} d \omega,
$$

where the target $\tau \in L^{2}(\Omega)$ and $N>0$ are given, as well as the set of feasible controls $K$. We assume that $K$ is a nonempty, closed and convex subset of $L^{s}(\Omega)$. The optimal control problem is

$$
\operatorname{Min}_{u} J_{\tau}\left(u, y_{u}\right) ; \text { subject to } u \in K
$$

As state and control spaces we choose

$$
Y:=W^{2, s}(\Omega) \cap W_{0}^{1, s}(\Omega)=\left\{y \in W^{2, s}(\Omega) ; y=0 \text { on } \partial \Omega\right\}, \quad U:=L^{s}(\Omega) .
$$

We denote $S\left(P_{\tau}\right)$ the set of solutions of $\left(P_{\tau}\right)$, and $\operatorname{val}\left(P_{\tau}\right)$ the value of $\left(P_{\tau}\right)$, i.e. $\inf \left\{J_{\tau}\left(u, y_{u}\right)\right.$; $u \in K\}$. In order to obtain existence of solutions, we will use the coercivity hypothesis

$$
\text { Either } n \leq 3 \text { or } K \text { is bounded in } L^{s}(\Omega) \text {. }
$$

This hypothesis implies that a minimizing sequence $\left\{u_{k}\right\}$ of controls for problem $\left(P_{\tau}\right)$ is bounded in $L^{s}(\Omega)$. By " $\stackrel{w}{\rightarrow}$ " we denote the weak convergence. Using standard techniques, we obtain the following result:

Theorem 2.1 Problem has (at least) one solution whenever the coercivity hypothesis 2.5 holds.

Proof.Consider a minimizing sequence $\left\{u_{k}\right\}$ of controls for problem $\left(P_{\tau}\right)$. By $(2.5)$, this sequence is bounded in $L^{s}(\Omega)$, and the sequence of associated states $\left\{y_{k}\right\}$ is bounded in $W^{2, s}(\Omega)$. Taking a subsequence if necessary, we may assume that $u_{k} \stackrel{w}{\rightarrow} \bar{u}$ in $L^{s}(\Omega), y_{k} \stackrel{w}{\rightarrow} \bar{y}$ in $W^{2, s}(\Omega)$ and $y_{k} \rightarrow \bar{y}$ in $C_{0}(\Omega)$. Passing to the limit in the state equation (2.1), we obtain that $\bar{y}$ is the state associated with $\bar{u}$. As $J_{\tau}$ is convex and continuous, and therefore weakly l.s.c. $L^{2}(\Omega) \times L^{s}(\Omega) \rightarrow \mathbb{R}$, we obtain $J_{\tau}(\bar{y}, \bar{u}) \leq \lim _{k} J_{\tau}\left(y_{k}, u_{k}\right)=\operatorname{val}\left(P_{\tau}\right)$. Finally, as $K$ is convex and closed in $L^{s}(\Omega)$, and therefore weakly closed, we have $\bar{u} \in K$. Therefore $\bar{u}$ is solution of $\left(P_{\tau}\right)$.

The adjoint equation is defined as

$$
\left\{\begin{aligned}
-\Delta p+\phi^{\prime}\left(y_{u}\right) p & = & y_{u}-\tau & \text { in } \Omega \\
p & = & 0 & \text { on } \partial \Omega .
\end{aligned}\right.
$$

$\mathrm{RR} \mathrm{n}^{\circ} 3014$ 
Its unique solution $p_{u} \in H^{2}(\Omega)$ is called the adjoint state associated with $u$. Set

$$
F(u):=J_{\tau}\left(u, y_{u}\right) .
$$

Using Green's formula and classical differential calculus arguments (e.g. [15]) we may check that $u \rightarrow F(u)$ is a $C^{2}$ mapping: $L^{s}(\Omega) \rightarrow Y$, with derivative

$$
F^{\prime}(u)=p_{u}+N u \text {. }
$$

We recall that, if $\mathcal{K}$ is a convex subset of a Banach space $\mathcal{X}$, and $x \in \mathcal{K}$, the cone of feasible directions $\mathcal{R}_{\mathcal{K}}$, and the tangent and normal cones $T_{\mathcal{K}}$ and $N_{\mathcal{K}}$, are defined as

$$
\begin{aligned}
\mathcal{R}_{\mathcal{K}}(x) & :=\{y \in \mathcal{X} ; \exists \sigma>0 ; x+\sigma y \in \mathcal{K}\} \\
T_{\mathcal{K}}(x) & :=\{y \in \mathcal{X} ; \exists x(\sigma)=x+\sigma y+o(\sigma) \in \mathcal{K}, \sigma \geq 0\} \\
N_{\mathcal{K}}(x) & :=\left\{x^{*} \in \mathcal{X} ;\left\langle x^{*}, y-x\right\rangle \leq 0, \forall y \in \mathcal{K}\right\}
\end{aligned}
$$

with the convention that $N_{\mathcal{K}}(x)=\emptyset$ if $x \notin \mathcal{K}$. The statement below is a standard result of optimization theory $([14,15])$ :

Proposition 2.1 If $u$ is a local solution of $\left(P_{\tau}\right)$ (in $L^{s}(\Omega)$ ), then it satisfies the first-order optimality condition

$$
F^{\prime}(u)+N_{K}(u) \ni 0 .
$$

Note that $F^{\prime}(u)$ belongs in principle to the space $U^{\prime}=L^{s^{\prime}}(\Omega)$, with $1 / s+1 / s^{\prime}=1$. However, from $u \in L^{s}(\Omega), p_{u} \in H^{2}(\Omega)$ and (2.7), we get $F^{\prime}(u) \in L^{2}(\Omega)$. This allows to obtain a characterization of the optimality system in terms of a projection:

Lemma 2.1 the optimality system (2.8) is equivalent to

$$
u=P_{K}\left(-N^{-1} p_{u}\right),
$$

where $P_{K}$ is the orthogonal projection in $L^{2}(\Omega)$ onto $K$.

Proof.Assume that (2.8) holds. As $L^{s}(\Omega) \subset L^{2}(\Omega), K$ is a convex (not necessarily closed) subset of $L^{2}(\Omega)$. Let $v \in K$. Then by (2.8), $\left\langle u+N^{-1} p_{u}, v-u\right\rangle_{2} \geq 0$ (note that this scalar product makes sense as $F^{\prime}(u) \in L^{s}(\Omega) \subset L^{2}(\Omega)$ ). Therefore

$$
\left\|v+N^{-1} p_{u}\right\|_{2}^{2}=\left\|u+N^{-1} p_{u}\right\|_{2}^{2}+2\left\langle u+N^{-1} p_{u}, v-u\right\rangle_{2}+\|v-u\|_{2}^{2} \geq\left\|u+N^{-1} p_{u}\right\|_{2}^{2} .
$$

This implies (2.9). The converse implication is an easy consequence of the above equality.

Using lemma 2.1, and assuming specific properties of $K$, some further properties of the optimal control may be derived. Assume for instance that $K$ is the set

$$
K_{a, b}:=\left\{u \in L^{s}(\Omega) ; a \leq u(\omega) \leq b, \text { a.e. } \omega \in \Omega\right\},
$$

INRIA 
with

$$
-\infty \leq a<b \leq+\infty
$$

Then the projection onto $K_{a, b}$ in $L^{2}(\Omega)$ coincides with the one in $L^{s}(\Omega)$ :

$$
P_{K_{a, b}}(u)(\omega)=\max (a, \min (u(\omega), b)), \text { a.e. } \omega \in \Omega .
$$

It is known that $W^{1, s}(\Omega)$ is a Banach lattice, whence the (punctual) maximum is a continuous operator in $W^{1, s}(\Omega)$ (e.g. [12, Chapter 7]). As $p_{u} \in W^{1, s}(\Omega)$, it follows from (2.10) that $u \in W^{1, s}(\Omega)$. Therefore $\Delta y_{u} \in W^{1, s}(\Omega)$, and if $\partial \Omega$ is of class $C^{3}$, then $y_{u} \in W^{3, s}(\Omega)$ [1]. Therefore the following result holds:

Lemma 2.2 Assume that the hypothesis of theorem 2.1 holds. If $K$ is of the form $K_{a, b}$ defined in (2.10), and $u$ satisfies the optimality system (2.8), then $u \in W^{1, s}(\Omega)$ and, if in addition $\partial \Omega$ is of class $C^{3}$, then $y_{u} \in W^{3, s}(\Omega)$.

\subsection{Second-order necessary and sufficient conditions}

With $u \in S\left(P_{\tau}\right)$ is associated the critical cone

$$
C(u):=\left\{v \in U ; F^{\prime}(u) v=0 ; v \in T_{K}(u)\right\} .
$$

This is the set of directions that are tangent to the feasible set, and along which, up to the first order term, the cost function does not increase. The second derivative of $u \rightarrow F(u)$ in the direction $v \in U$ is easily checked to be

$$
F^{\prime \prime}(u)(v, v)=\int_{\Omega}\left[N v(\omega)^{2} d \omega+\left(1-p_{u}(\omega) \phi^{\prime \prime}(y(\omega))\right) z_{v}(\omega)^{2}\right] d \omega,
$$

where $z_{v}$ is solution of $(2.4)$.

We recall the concept of polyhedricity, originally defined in a Hilbert space framework $[20,13]$ (see an extension of this concept in $[6]$ ).

Definition 2.1 Let $K$ be a closed convex subset of a Banach space $X, x_{0} \in K$ and $x^{*} \in$ $N_{K}\left(x_{0}\right)$. We say that $K$ is polyhedric at $x$ for the normal direction $x^{*}$ if

$$
T_{K}\left(x_{0}\right) \cap\left(x^{*}\right)^{\perp}=\overline{\mathcal{R}_{K}\left(x_{0}\right) \cap\left(x^{*}\right)^{\perp}} .
$$

If $K$ is polyhedric at each $x_{0} \in K$ for all $x^{*} \in N_{K}\left(x_{0}\right)$, we say that $K$ is polyhedric.

Definition 2.2 We define the second-order necessary optimality condition as

$$
\forall v \in C(u), \quad F^{\prime \prime}(u)(v, v) \geq 0 .
$$

Theorem 2.2 Let $u$ be a local solution of $S\left(P_{\tau}\right)$. If $K$ is polyhedric, then $u$ satisfies the second-order necessary condition (2.15).

$\mathrm{RR} \mathrm{n}^{\circ} 3014$ 
Proof.Let $v \in \mathcal{R}_{K}\left(x_{0}\right) \cap F^{\prime}(u)^{\perp}$. Then as $u+t v$ is feasible for $t>0$ small enough, and $F^{\prime}(u) v=0$, we have

$$
0 \leq 2 \lim _{t \downarrow 0} t^{-2}(F(u+t v)-F(u))=F^{\prime \prime}(u)(v, v) .
$$

By polyhedricity, the set of such $v$ is dense in the critical cone, while $v \rightarrow F^{\prime \prime}(u)(v, v)$ is a continuous function $L^{s}(\Omega) \rightarrow \mathbb{R}$. The second order necessary condition follows.

Having motivated by this theorem the importance of polyhedricity, we check that the concept applies to $K_{a, b}$ :

Proposition 2.2 The set $K_{a, b}$ is polyhedric in $L^{s}(\Omega)$ for all $s \in(2, \infty)$, with $-\infty \leq a<$ $b \leq+\infty$.

Proof.Let $v \in C(u)$. For $\varepsilon>0$, set $v_{\varepsilon}:=\varepsilon^{-1}\left(P_{K}(u+\varepsilon v)-u\right)$. Obviously $v_{\varepsilon} \in \mathcal{R}_{K}(u)$. Also $F^{\prime}(u) v_{\varepsilon}=0$, as by $(2.8)$ and $(2.10), F^{\prime}(u)(\omega)=0$ whenever $v_{\varepsilon}(\omega) \neq 0$, a.e. on $\Omega$. Therefore $v_{\varepsilon}$ is a critical direction. As $v \in T_{K}(u)$, we have that $v_{\varepsilon} \rightarrow v$ when $\varepsilon \downarrow 0$. Polyhedricity of $K_{a, b}$ follows.

We turn now to the sufficient conditions for optimality.

Definition 2.3 We say the $u$ satisfies the standard quadratic growth condition if

$$
\exists \alpha>0 ; \quad F(v) \geq F(u)+\alpha\|v-u\|_{s}^{2}+o\left(\|v-u\|_{s}^{2}\right), \quad \forall v \in K,
$$

and the weak quadratic growth condition if

$$
\begin{aligned}
& \exists \varepsilon>0 ; \quad \exists \alpha>0 ; \quad \text { if } v \in K, \quad\|v-u\|_{s} \leq \varepsilon ; \\
& F(v) \geq F(u)+\alpha\|v-u\|_{2}^{2}+o\left(\|v-u\|_{2}^{2}\right) .
\end{aligned}
$$

Definition 2.4 Let $u \in K$ satisfy the optimality system (2.8). We say that $u$ satisfies the standard second-order sufficient optimality condition if

$$
\exists \alpha>0 ; \quad \forall v \in C(u), \quad F^{\prime \prime}(u)(v, v) \geq \alpha\|v\|_{s}^{2},
$$

and the weak second-order sufficient optimality condition if

$$
\exists \alpha>0 ; \quad \forall v \in C(u), \quad F^{\prime \prime}(u)(v, v) \geq \alpha\|v\|_{2}^{2} .
$$

Note that the strong conditions imply the weak ones, and both are equivalent if $n \leq 3$. We consider also the following relation, very close to the second order necessary condition:

$$
\forall v \in C(u) \backslash\{0\}, \quad F^{\prime \prime}(u)(v, v)>0 .
$$

We first discuss the case when $n \leq 3$. 
Theorem 2.3 Assume that $n \leq 3$ (whence $s=2$ ). If $K$ is polyhedric, then the strong quadratic growth condition (2.16), the second-order sufficient condition (2.18) and (2.20) are equivalent.

Proof.We prove that $(2.16) \Rightarrow(2.18) \Rightarrow(2.20) \Rightarrow(2.16)$. Assume that $(2.16)$ holds. Let $v \in$ $\left.\mathcal{R}_{K}\left(x_{0}\right) \cap\left(F^{\prime}(u)\right)\right)^{\perp}$. By $(2.16), F(u+t v)-F(u) \geq \alpha t^{2}\|v\|_{2}^{2}+o\left(t^{2}\right)$, whence $F^{\prime \prime}(u)(v, v) \geq$ $2 \alpha\|v\|_{2}^{2}$. The second order sufficient condition follows then by polyhedricity.

That (2.18) implies (2.20) is trivial. Assume now that (2.20) holds, while (2.16) is not satified. Then there exists a sequence $u_{k} \rightarrow u$ in $L^{2}(\Omega)$ such that

$$
F\left(u_{k}\right)<F(u)+\frac{1}{k}\left\|u_{k}-u\right\|_{2}^{2} .
$$

Extracting if necessary a subsequence, we may write $u_{k}=t_{k} v_{k}$, with $t_{k} \in \mathbb{R}_{+*}, t_{k} \downarrow 0$, $\left\|v_{k}\right\|_{2}=1$, and $v_{k} \stackrel{w}{\rightarrow} \bar{v}$ in $L^{2}(\Omega)$. Using the second order expansion

$$
F\left(u_{k}\right)=F(u)+t_{k} F^{\prime}(u) v_{k}+\frac{t_{k}^{2}}{2} F^{\prime \prime}(u)\left(v_{k}, v_{k}\right)+o\left(t_{k}^{2}\right),
$$

and (2.21), we obtain $F^{\prime}(u) \bar{v}=0$ and $\left(\operatorname{as~} F^{\prime}(u) v_{k} \geq 0\right) \lim \sup _{k} F^{\prime \prime}(u)\left(v_{k}, v_{k}\right) \leq 0$. As $v_{k}$ belongs to the weakly closed set $T_{K}(u)$, it follows that $\bar{v}$ is a critical direction. By (2.13), we may write

$$
F^{\prime \prime}(u)(v, v)=N\|v\|_{2}^{2}+Q_{u}(v),
$$

where $Q_{u}(v)$ is a weakly continuous quadratic form. Therefore, $F^{\prime \prime}(u)$ is weakly l.s.c., whence $F^{\prime \prime}(u) \bar{v} \bar{v} \leq 0$. Combining with (2.23), we obtain

$$
Q_{u}(\bar{v})=\lim Q_{u}\left(v_{k}\right) \leq-N \liminf \left\|v_{k}\right\|_{2}^{2}=-N .
$$

It follows that $\bar{v}$ cannot be 0 . This contradicts (2.20).

The extension of this statement when $n>3$ is based on the following result:

Lemma 2.3 Whenever $v$ remains in a bounded subset of $L^{s}(\Omega)$, the following relations holds:

$$
\begin{aligned}
y_{u+v} & =y_{u}+y_{u}^{\prime} v+\frac{1}{2} y_{u}^{\prime \prime}(v, v)+o\left(\|v\|_{2}^{2}\right) \text { in } H^{2}(\Omega), \\
F(u+v) & =F(u)+F^{\prime}(u) v+\frac{1}{2} F^{\prime \prime}(u)(v, v)+o\left(\|v\|_{2}^{2}\right) .
\end{aligned}
$$

Proof.Step 1. Consider the case when $\phi$ and $\phi^{\prime}$ are globally Lipschitz. Then $y \rightarrow$ $-\Delta y+\phi(y)$ is a $C^{2}$ mapping: $H^{2}(\Omega) \rightarrow L^{2}(\Omega)$, and the linearized equation (2.4) has a unique solution $z \in H^{2}(\Omega)$. By the implicit function theorem, (2.25) holds. Relation (2.26) follows from the composition of $u \rightarrow y_{u}$ and $\left(y_{u}, u\right) \rightarrow J_{\tau}\left(y_{u}, u\right)$.

Step 2. Fix $r>\|u\|_{s}$. By (2.2) and (2.3), there exists $M>0$ such that $\left\|y_{v}\right\|_{\infty} \leq M$ whenever $\|v\|_{s} \leq r$. Therefore $y_{v}$ is determined only by the the value of $\phi$ over $[-M,+M]$. We may modify the value of $\phi$ outside $[-M,+M]$ so that the new $\phi$ is nondecreasing and is globally Lipschitz as well as its first derivative. Combining with step 1, we obtain the conclusion.

RR $n^{\circ} 3014$ 
Theorem 2.4 If $K$ is polyhedric, then the weak quadratic growth condition (2.17), the second-order sufficient condition (2.19) and (2.20) are equivalent.

Proof.The proof is identical to the one of theorem 2.3, relation (2.22) being consequence of lemma 2.3.

We end this section by some remarks.

The above problem may be related to the notion of tangent quadratic problem, defined as follows (see the related discussion in [4]):

$$
\operatorname{Min}_{v} F^{\prime}(u) v+\frac{1}{2} F^{\prime \prime}(u)(v, v) ; \quad v \in T_{K}(u) .
$$

Assume that $u$ satisfies the first-order optimality system. Problems $\left(P_{\tau}\right)$ and $\left(Q_{\tau}\right)$ have the same critical cone and same second-order expansion of the cost function. In addition, the tangent quadratic problem is always polyhedric at $v=0$. Therefore, if $K$ is polyhedric, then the second order necessary (resp. sufficient) conditions for optimality coincide for problems $\left(P_{\tau}\right)$ and $\left(Q_{\tau}\right)$. By theorem 2.4, it follows that the weak quadratic growth condition holds for $\left(P_{\tau}\right)$ iff it holds for $\left(Q_{\tau}\right)$.

If $\bar{u}$ is a local solution of $\left(P_{\tau}\right)$, then it is a local solution of $\left(Q_{\tau}\right)$, that is isolated if and anly if the quadratic growth condition holds. That is, if the quadratic growth condition does not holds, then there exists a nonzero solution of the tangent quadratic problem $\left(Q_{\tau}\right)$. This is similar to the Jacobi conditions in the calculus of variations.

have the following alternative : either the quadratic growth condition is satisfied or $v=0$ It is not difficult to see that the Hessian of $F$ is a Legendre form [14] in the sense below:

Definition 2.5 We say that a quadratic $Q$ form on a Hilbert space $X$ is a Legendre form if $Q$ is weakly l.s.c. and, whenever a sequence $\left\{x_{k}\right\} \subset X$ satisfies $x_{k} \stackrel{w}{\rightarrow} x$ and $Q\left(x_{k}\right) \rightarrow Q(x)$, then $x_{k} \rightarrow x$.

Theorems 2.2 and 2.3 may be obtained by specializing general statements [8], where it is assumed that the set of feasible points is polyhedric, that the cost function is of class $C^{2}$ and, for the second order sufficient condition, that the Hessian of the cost function is a Legendre form. Theorem 2.4 is new. The two norms approach is a classical idea in the calculus of variations, the spaces used being $L^{2}(0, T)$ and $L^{\infty}(0, T)$, but the classical results concerning second order conditions essentially deal with unconstrained problems. The extension to polyhedric constraint sets is discussed in $[16,17,18]$, assuming stronger second order conditions, that extend the strong regularity condition of [21]. Related results, in the context of optimal control of partial differential equation, may be found in $[9,10]$. These papers consider more general nonlinearities and, therefore, use the two norms approach with spaces $L^{2}(\Omega)$ and $L^{\infty}(\Omega)$. Note also that there is no characterization of quadratic growth in these papers. 


\section{Sensitivity analysis}

We discuss in this section the dependance of a local solution of $\left(P_{\tau}\right)$ with respect to the parameter $\tau$. We start with a stability result. We introduce the notations

$$
F(u, \tau):=J_{\tau}(y, u) ; \quad \operatorname{val}(\tau):=\inf F(u, \tau) ; u \in K .
$$

Denote by $z_{v}$ the solution of (2.4) for $(u, y)=(\bar{u}, \bar{y})$, and let $\bar{p}$ the the adjoint state associated with $(\bar{u}, \bar{y})$. Let $\eta \in L^{2}(\Omega)$. Then

$$
F^{\prime \prime}(\bar{u}, \bar{\tau})((v, \eta),(v, \eta))=\int_{\Omega}\left(N v(\omega)^{2}+\left(z_{v}(\omega)-\eta(\omega)\right)^{2}-\bar{p}(\omega) \phi^{\prime \prime}(\bar{y}(\omega)) z_{v}(\omega)^{2}\right) d \omega .
$$

We introduce the subproblem

$$
\operatorname{Min}_{v} \quad F^{\prime \prime}(\bar{u}, \tau)((v, \eta),(v, \eta)) \quad \text { subject to } v \in C(\bar{u}) .
$$

Under the coercivity hypothesis (2.5), this problem has a finite value and a nonempty set of solutions.

Theorem 3.1 If the coercivity hypothesis (2.5) holds, then

(i) If $\tau_{k} \rightarrow \bar{\tau}$ in $L^{2}(\Omega)$ and $u_{k} \in S\left(P_{\tau_{k}}\right)$, then there exists $\bar{u} \in S\left(P_{\bar{\tau}}\right)$ such that, extracting a subsequence if necessary, $\bar{u}$ is the weak limit in $L^{s}(\Omega)$ and strong limit in $L^{2}(\Omega)$ of a subsequence of $\left\{u_{k}\right\}$. If in addition $\bar{u}$ satisfies the weak second order sufficient condition (2.19) (for problem $\left(P_{\bar{\tau}}\right)$ ), then for the associated subsequence we have

$$
\left\|u_{k}-\bar{u}\right\|_{2}=O\left(\left\|\tau_{k}-\bar{\tau}\right\|_{2}\right) .
$$

(ii) If $\tau_{k}=\bar{\tau}+t_{k} \eta$, then the above mentioned $\bar{u}$ is solution of

$$
\operatorname{Min}_{u}\left\langle\bar{\tau}-y_{u}, \eta\right\rangle_{2} ; u \in S\left(P_{\bar{\tau}}\right) .
$$

If in addition $\bar{u}$ satisfies (2.19), then for the considered subsequence we have the second order expansion of the cost

$$
\operatorname{val}\left(t_{k}\right)=\operatorname{val}\left(P_{\bar{\tau}}\right)+t_{k}\left\langle\bar{\tau}-y_{\bar{u}}, \eta\right\rangle_{2}+\frac{t_{k}^{2}}{2} \operatorname{val}(S P)+o\left(t_{k}^{2}\right),
$$

and if $v$ is a weak limit-points of $\left(u_{k}-\bar{u}\right) / t_{k}$ in $L^{2}(\Omega)$ (there exist some by (i)), then $v$ is a strong limit point of $\left(u_{k}-\bar{u}\right) / t_{k}$ in $L^{2}(\Omega)$ and is solution of $(S P)$.

Proof.(i) That $\bar{u}$ is the weak limit in $L^{s}(\Omega)$ and strong limit in $L^{2}(\Omega)$ of a subsequence may be proved by arguments similar to those in the proof of theorem 2.1 , the strong convergence in $L^{2}(\Omega)$ being a consequence of the convergence of $\operatorname{val}\left(\tau_{k}\right)$ toward $\operatorname{val}(\bar{\tau})$.

RR $\mathrm{n}^{\circ} 3014$ 
Assume now that $\bar{u}$ satisfies the second order sufficient condition (2.19) for problem $\left(P_{\bar{\tau}}\right)$. We have

$$
\operatorname{val}(\tau) \leq F(\bar{u}, \tau)=F(\bar{u}, \bar{\tau})+F_{\tau}^{\prime}(\bar{u}, \bar{\tau})(\tau-\bar{\tau})+\frac{1}{2}\|\tau-\bar{\tau}\|_{2}^{2} .
$$

Let $u_{\tau} \in S\left(P_{\tau}\right)$. Then

$$
\begin{aligned}
\operatorname{val}(\tau)= & F\left(u_{\tau}, \tau\right), \\
= & F(\bar{u}, \bar{\tau})+F^{\prime}(\bar{u}, \bar{\tau})\left(u_{\tau}-\bar{u}, \tau-\bar{\tau}\right)+ \\
& \frac{1}{2} F^{\prime \prime}(\bar{u}, \bar{\tau})\left(\left(u_{\tau}-\bar{u}, \tau-\bar{\tau}\right),\left(u_{\tau}-\bar{u}, \tau-\bar{\tau}\right)\right)+o\left(\left\|u_{\tau}-\bar{u}\right\|_{2}^{2}+\|\tau-\bar{\tau}\|_{2}^{2}\right) .
\end{aligned}
$$

Combining both relations, and noticing that the first order optimality system (2.8) implies $F^{\prime}(\bar{u}, \bar{\tau})\left(u_{\tau}-\bar{u}, \tau-\bar{\tau}\right) \geq F_{\tau}^{\prime}(\bar{u}, \bar{\tau})(\tau-\bar{\tau})$, we obtain

$$
F^{\prime \prime}(\bar{u}, \bar{\tau})\left(\left(u_{\tau}-\bar{u}, \tau-\bar{\tau}\right),\left(u_{\tau}-\bar{u}, \tau-\bar{\tau}\right)\right)+o\left(\left\|u_{\tau}-\bar{u}\right\|_{2}^{2}+\|\tau-\bar{\tau}\|_{2}^{2}\right) \leq\|\tau-\bar{\tau}\|_{2}^{2} .
$$

Consider the subsequence $u_{k}:=u_{\tau_{k}}$ converging to $\bar{u}$ in $L^{s}(\Omega)$ weak and $L^{2}(\Omega)$ strong. If (extracting if necessary a subsequence) $\left\|u_{k}-\bar{u}\right\|_{2} /\left\|\tau_{k}-\bar{\tau}\right\|_{2} \rightarrow+\infty$, it follows from the above relation that

$$
F_{u^{2}}^{\prime \prime}(\bar{u}, \bar{\tau})\left(u_{k}-\bar{u}, u_{k}-\bar{u}\right) \leq o\left(\left\|u_{k}-\bar{u}\right\|_{2}^{2}\right) .
$$

Let $\bar{v}$ be a weak limit point in $L^{2}(\Omega)$ of $v_{k}:=\left(u_{k}-\bar{u}\right) /\left\|u_{k}-\bar{u}\right\|_{2}$. By arguments similar to those of the proof of theorem 2.3 we find that $F_{u^{2}}^{\prime \prime}(\bar{v}, \bar{v}) \leq 0, \bar{v} \in C(\bar{u})$ and $\bar{v} \neq 0$. This contradicts the second order sufficient optimality condition (2.20), that is equivalent to (2.19) by theorem 2.4 .

(ii) Let $u \in S\left(P_{\bar{\tau}}\right)$. As

$$
\operatorname{val}\left(\tau_{k}\right) \leq F\left(u, \tau_{k}\right)=F(u, \bar{\tau})+t_{k} F_{\tau}^{\prime}(u, \bar{\tau}) \eta+\frac{t_{k}^{2}}{2}\|\eta\|_{2}^{2},
$$

we have

$$
\operatorname{val}_{+}^{\prime}(\bar{\tau}, \eta):=\limsup t_{k}^{-1}\left(\operatorname{val}\left(\bar{\tau}+t_{k} \eta\right)-\operatorname{val}(\bar{\tau})\right) \leq \inf _{u \in S\left(P_{\bar{\tau}}\right)} F_{\tau}^{\prime}(u, \bar{\tau}) \eta .
$$

On the other hand, as $u_{k} \in S\left(P_{\tau_{k}}\right)$ converges to $\bar{u}$ in $L^{s}(\Omega)$ weakly, and $F_{\tau}^{\prime}\left(u_{k}, \bar{\tau}\right)=\bar{\tau}-y_{u_{k}}$, we have $F_{\tau}^{\prime}\left(u_{k}, \bar{\tau}\right) \rightarrow F_{\tau}^{\prime}(\bar{u}, \bar{\tau})$ in $L^{2}(\Omega)$. Therefore

$$
\begin{aligned}
\operatorname{val}\left(\tau_{k}\right) & =F\left(u_{k}, \tau_{k}\right)=F\left(u_{k}, \bar{\tau}\right)+t_{k} F_{\tau}^{\prime}\left(u_{k}, \bar{\tau}\right) \eta+o\left(t_{k}\right) \\
& \geq F(\bar{u}, \bar{\tau})+t_{k} F_{\tau}^{\prime}(\bar{u}, \bar{\tau}) \eta+o\left(t_{k}\right) .
\end{aligned}
$$

Relation (3.28) follows.

Assume now that $\bar{u}$ satisfies (2.19). Let $v \in \mathcal{R}_{K}(\bar{u}) \cap C(\bar{u})$. Expanding $F$, we find that

$$
\operatorname{val}(\bar{\tau}+t \eta) \leq F(\bar{u}+t v, \bar{\tau}+t \eta)=\operatorname{val}(\bar{\tau})+t F_{\tau}^{\prime}(\bar{u}, \bar{\tau}) \eta+\frac{t^{2}}{2} F^{\prime \prime}(\bar{u}, \bar{\tau})((v, \eta),(v, \eta))+o\left(t^{2}\right)
$$


As $F^{\prime \prime}(\bar{u}, \bar{\tau})((v, \eta),(v, \eta))$ is nothing but the cost function in $(S P)$, using polyhedricity, we deduce that

$$
\limsup \frac{\operatorname{val}(\bar{\tau}+t \eta)-\operatorname{val}(\bar{\tau})-t F_{\tau}^{\prime}(\bar{u}, \bar{\tau}) \eta}{t^{2} / 2} \leq \operatorname{val}(S P) .
$$

On the other hand, by (i), the sequence $v_{k}:=\left(u_{k}-\bar{u}\right) / t_{k}$ has at least one weak limit-point $v$ in $L^{2}(\Omega)$. From

$$
F\left(u_{k}, \tau_{k}\right)=F(\bar{u}, \bar{\tau})+t_{k} F^{\prime}(\bar{u}, \bar{\tau})\left(v_{k}, \eta\right)+\frac{t_{k}^{2}}{2} F^{\prime \prime}(\bar{u}, \bar{\tau})\left(v_{k}, \eta\right)\left(v_{k}, \eta\right)+o\left(t_{k}^{2}\right),
$$

and the lower semi continuity of $F^{\prime \prime}$, we deduce that $v$ is a critical direction, and

$$
\begin{aligned}
F\left(u_{k}, \tau_{k}\right) & \geq F(\bar{u}, \bar{\tau})+t_{k} F_{\tau}^{\prime}(\bar{u}, \bar{\tau}) \eta+\frac{t_{k}^{2}}{2} F^{\prime \prime}(\bar{u}, \bar{\tau})((v, \eta),(v, \eta))+o\left(t_{k}^{2}\right), \\
& \geq F(\bar{u}, \bar{\tau})+t_{k} F_{\tau}^{\prime}(\bar{u}, \bar{\tau}) \eta+\frac{t_{k}^{2}}{2} \operatorname{val}(S P)+o\left(t_{k}^{2}\right) .
\end{aligned}
$$

Combining with (3.32), we obtain the second-order expansion of the cost, as well as

$$
F^{\prime \prime}(\bar{u}, \bar{\tau})\left(v_{k}, \eta\right)\left(v_{k}, \eta\right) \rightarrow \operatorname{val}(S P) .
$$

From the expression of $F^{\prime \prime}$, the latter implies that, for the considered subsequence, $v_{k} \rightarrow v$ strongly in $L^{2}(\Omega)$.

Remark. Note that if $\bar{u}$ is a local solution of $S\left(P_{\bar{\tau}}\right)$ satisfying the weak quadratic growth condition (2.17), then there exists a closed neighborhood $\mathcal{U}$ of $u$ in $U$ such that the local problem

$\left(P_{\tau, \mathcal{U}}\right) \quad \operatorname{Min}_{u} J_{\tau}\left(u, y_{u}\right) ;$ subject to $(2.1), u \in \mathcal{U}$ and $u \geq 0$ on $\Omega$,

has, when $\tau=\bar{\tau}$, the unique solution $\bar{u}$. The additional constraint $u \in \mathcal{U}$ is not active for $\tau$ close to $\bar{\tau}$. We can conduct a similar perturbation analysis for the localized problem. If in addition problem $(S P)$ has a unique solution $\bar{v}$, we obtain that if $u(t) \in S\left(P_{\bar{\tau}+t \eta, \mathcal{U}}\right)$, then $u(t)=\bar{u}+t \bar{v}+o(t)$, i.e. the path $u(t)$ has a right directional derivative $\bar{v}$.

If $n \leq 3$, theorem 3.1 is a consequence of the results in [6]. In that case it improves the results in [23] where stronger second order conditions are assumed. The result may be generalized in the following way: given two control spaces $U_{1}$ and $U_{2}$, with $U_{1} \subset U_{2}$, and a polyhedric convex feasible set $K \subset U_{1}$, we have to assume that in bounded subsets of $U_{2}$, the cost function has a second order expansion in terms of the norm of $U_{1}$. Then, if the corresponding weak second order sufficient condition applies, a similar perturbation analysis may be conducted.

Related results are $[16,23]$ where the derivative of solution is computed under stronger second order conditions, that on the other hand guarantee the uniqueness of the solution of the perturbed problem.

$\mathrm{RR} \mathrm{n}^{\circ} 3014$ 


\section{Generalization of the previous results: beyond poly- hedricity}

We denote $L_{\ell}^{s}(\Omega):=L^{s}(\Omega) \times \cdots \times L^{s}(\Omega)(\ell$ times $)$, and $\|\cdot\|_{s}$ its associated product norm. We adopt similar conventions for $H_{\ell}^{s}(\Omega)$ and $W_{\ell}^{m, s}(\Omega)$. We consider a problem of the form

$\operatorname{Min} F(u, \tau) ; u \in K$,

where $K$ is a closed convex subset of $L_{\ell}^{s}(\Omega)$, i.e. we have now $u(\omega) \in \mathbb{R}^{\ell}$. All statements of this section assume the following on $F$ :

\section{Assumption A.}

(i) The function $F$ is $C^{2}: L_{\ell}^{s}(\Omega) \rightarrow \mathbb{R}$.

(ii) If $u \in L_{\ell}^{\infty}(\Omega)$, then $F_{u^{2}}^{\prime \prime}$ is a Legendre form on $L_{\ell}^{2}(\Omega)$ and $F^{\prime}(u) \in H_{\ell}^{2}(\Omega)$.

These hypotheses are satisfied in the case of problem $\left(P_{\tau}\right)$ studied before, with $\ell=1$. Let us give an example that motivates the study of the case $\ell>1$. Consider the coupled system

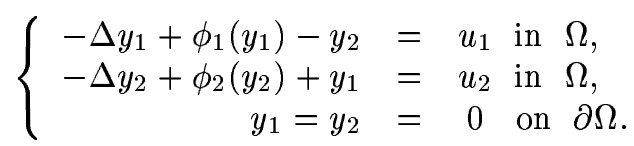

Here $y(\omega)=\left(y_{1}(\omega), y_{2}(\omega)\right) \in \mathbb{R}^{2}$, while $\phi_{1}$ and $\phi_{2}$ are $C^{2}$ nondecreasing mappings $\mathbb{R} \rightarrow \mathbb{R}$. In the sequel, by $|\cdot|$ we denote the Euclidean norm as well as absolute value of scalars, and by $\langle\cdot, \cdot\rangle$ the associated scalar product. We denote by $y_{u}$ the solution of this system (whose existence and uniqueness is discussed in the following lemma) and consider the cost function

$$
F(u, \tau):=\frac{1}{2} \int_{\Omega}|y(\omega)-\tau(\omega)|^{2} d \omega+\frac{N}{2} \int_{\Omega}|u(\omega)|^{2} d \omega .
$$

Lemma 4.1 For each $u \in L_{\ell}^{s}(\Omega)$, with $s \geq 2$, system (4.33) has a unique solution $y_{u} \in$ $W_{\ell}^{2, s}(\Omega)$. In addition

(i) the mapping $u \rightarrow y_{u}$ is of class $C^{2}: L_{\ell}^{s}(\Omega) \rightarrow W_{\ell}^{2, s}(\Omega)$,

(ii) Assumption A is satisfied.

Proof.(i) We may assume for simplicity that $\phi_{1}(0)=\phi_{2}(0)=0$. Multiplying the first (resp. second) equation by $y_{1}$ (resp. $y_{2}$ ) and integrating over $\Omega$, we obtain the a priori estimate (i.e. estimate satisfied by any sufficiently smooth solution of (4.33)):

$$
\begin{aligned}
\int_{\Omega}\left(\left|\nabla y_{1}(\omega)\right|^{2}+\left|\nabla y_{2}(\omega)\right|^{2}\right) d \omega & \leq \int_{\Omega}\left(y_{1}(\omega) u_{1}(\omega)+y_{2}(\omega) u_{2}(\omega)\right) d x \\
& \leq\left\|y_{1}\right\|_{2}\left\|u_{1}\right\|_{2}+\left\|y_{2}\right\|_{2}\left\|u_{2}\right\|_{2} .
\end{aligned}
$$

By $a_{i}$ and $a_{i}^{\prime}, i=0,1, \cdots$, we denote constants depending on $\Omega$ only. Combining the above display with Poincaré's inequality, we obtain the estimate $\|y\|_{1,2} \leq a_{0}\|u\|_{2}$. With (4.33) it 
follows that $\|-\Delta y+\phi(y)\|_{2} \leq a_{0}^{\prime}\|u\|_{2}$, whence the $H_{\ell}^{2}(\Omega)$ estimate $\|y\|_{2,2} \leq a_{1}\|u\|_{2}$. Then we may use a bootstrapping argument: set $s_{1}:=2$. As, by $(2.2), H_{\ell}^{2}(\Omega) \subset L_{\ell}^{s_{2}}(\Omega)$ with continuous injection, with $1 / s_{2}=1 / s_{1}-2 / n$, we have if $s_{2} \leq s$

$$
\|-\Delta y+\phi(y)\|_{s_{2}} \leq a_{2}^{\prime}\left(\|y\|_{s_{2}}+\|u\|_{s_{2}}\right) \leq a_{2}^{\prime \prime}\|u\|_{s_{2}},
$$

whence the estimate $\|y\|_{2, s_{2}} \leq a_{2}\|u\|_{s_{2}}$. We may iterate while $s_{i} \leq s$. As $1 / s_{i+1}=1 / s_{i}-2 / n$, after a finite number of steps we get the final a priori estimate $\|y\|_{2, s} \leq a_{2}\|u\|_{s}$. The existence of a solution may be proved by standard fixed point arguments (e.g. [5]) while uniqueness is proved as follows: let $y$ and $\hat{y}$ two solutions, and set $z:=y-\hat{y}$. Then $z$ is solution of

$$
\left\{\begin{aligned}
-\Delta z_{1}+\psi_{1} z_{1}-z_{2} & =0 \text { in } \Omega \\
-\Delta z_{2}+\psi_{2} z_{2}+z_{1} & =0 \text { in } \Omega \\
z_{1}=z_{2} & =0 \text { on } \partial \Omega
\end{aligned}\right.
$$

where for $i=1,2$

$$
\psi_{i}(\omega):=\frac{\phi_{i}\left(y_{i}(\omega)\right)-\phi_{i}\left(\hat{y}_{i}(\omega)\right)}{z_{i}(\omega)} \text { if } z_{i}(\omega) \neq 0, \quad \phi_{i}^{\prime}\left(y_{i}(\omega)\right) \text { otherwise. }
$$

As $\psi_{i}$ is nonnegative, $i=1,2$, multiplying the first (resp. second) equation by $z_{1}$ (resp. $z_{2}$ ) we obtain

$$
\int_{\Omega}\left(\left|\nabla z_{1}(\omega)\right|^{2}+\left|\nabla z_{2}(\omega)\right|^{2}\right) d \omega \leq 0 .
$$

This with Poincaré's inequality, implies $z=0$, as desired.

(ii) The result is obtained by combining point (i) and the techniques of section 2 .

Consider the case when $K$ is defined by local constraints:

$$
K:=\left\{u \in L_{\ell}^{s}(\Omega) ; u(\omega) \in \underline{K}(\omega), \text { a.e. } \omega \in \Omega\right\},
$$

where $\underline{K}(\cdot)$ is a closed convex subset of $\mathbb{R}^{\ell}$, a.e. on $\Omega$. The following statement is a classical result [22].

Lemma 4.2 Let $K$ be a closed convex subset of $L_{\ell}^{s}(\Omega)$ satisfying (4.35), where $\underline{K}(\omega)$ is a closed and convex set, a.e. $\omega \in \Omega$. Then

(i) $T_{K}(u) \quad=\left\{v \in L_{\ell}^{s}(\Omega) ; v(\omega) \in T_{\underline{K}(\omega)}\right.$, a.e. $\left.\omega \in \Omega\right\}$;

(ii) $N_{K}(u) \quad=\left\{z \in L_{\ell}^{s^{\prime}}(\Omega) ; z(\omega) \in \bar{N}_{K(\omega)}\right.$, a.e. $\left.\omega \in \Omega\right\}$,

(iii) $T_{K}(u) \cap\left(u^{*}\right)^{\perp}=\left\{v \in T_{K}(u) ;\left\langle u^{*}(\omega), v(\omega)\right\rangle=0\right.$, a.e. $\left.\omega \in \Omega\right\}$.

We now study the case when $\underline{K}(\omega)$ is defined by a finite number of linear constraints. For the sequel it is useful to deal with the case of a variable set of constraints.

RR $\quad{ }^{\circ} 3014$ 
Proposition 4.1 Assume that

$$
\underline{K}(\omega)=\left\{x \in \mathbb{R}^{\ell} ;\left\langle a_{i}(\omega), x\right\rangle \leq b_{i}(\omega), i=J(\omega)\right\} .
$$

Here $J(\omega)$ is a finite set included in $\{1, \cdots, q\}, \omega \rightarrow J(\omega)$ is a measurable mapping, and $a_{i}(\omega)$ (resp. $\left.b_{i}(\omega)\right)$ belongs to $L_{\ell}^{\infty}(\Omega)$ (resp. $L_{q}^{\infty}(\Omega)$ ), $i=1, \cdots, q$. Then $K$ is a closed convex polyhedric subset of $L_{\ell}^{s}(\Omega)$.

Proof.It is easily checked that $K$ is a closed convex subset of $L_{\ell}^{s}(\Omega)$. By the above lemma, (4.36) applies. Let us prove that $K$ is polyhedric. Fix $u \in K$ and $u^{*} \in N_{K}(u)$, and let $v \in T_{K}(u) \cap\left(u^{*}\right)^{\perp}$. Given $\varepsilon>0$, set

$$
v_{\varepsilon}(\omega)=v(\omega) \text { if } u(\omega)+\varepsilon v(\omega) \in \underline{K}(\omega) ; 0 \text { otherwise, a.e. } x \in \Omega .
$$

From (4.36iii) it follows that $v_{\varepsilon} \in T_{K}(u) \cap\left(u^{*}\right)^{\perp}$. Also $\left|v_{\varepsilon}(\omega)\right| \leq|v(\omega)|$ and by (4.36i), $v_{\varepsilon}(\omega) \rightarrow v(\omega)$ a.e. on $\Omega$; Lebesgue's theorem implies that $v_{\varepsilon} \rightarrow v$ in $L_{\ell}^{s}(\Omega)$. The result follows.

We now extend our results to the case of nonlinear local constraints. We first give a formula for normal cones.

Lemma 4.3 Let $g: \mathbb{R}^{q} \rightarrow \mathbb{R}$ be a $C^{2}$ convex mapping with uniformly bounded first and second order derivatives, such that

$$
g\left(x_{0}\right)<0 \text { for some } x_{0} \in \mathbb{R}^{q},
$$

and that $\{x ; g(x) \leq 0\}$ is bounded. Assume that

$$
\underline{K}(\omega)=\underline{K}:=\left\{z \in \mathbb{R}^{\ell} ; g(z) \leq 0\right\},
$$

is independant of $\omega$. Let $u \in K$. Set

$$
I(\omega):=\left\{i=1, \cdots, q ; g_{i}(u(\omega))=0\right\} .
$$

Then

(i) $T_{K}(u)=\left\{v \in L_{\ell}^{s}(\Omega) ; g_{i}^{\prime}(u(\omega)) v(\omega) \leq 0, i \in I(\omega)\right.$ a.e. $\left.\omega \in \Omega\right\}$;

(ii) $N_{K}(u)=\left\{u^{*} \in L_{\ell}^{s^{\prime}}(\Omega) ; \exists \lambda \in L_{\ell}^{s^{\prime}}(\Omega) ; u^{*}(\omega)=\sum_{i=1}^{q} \lambda_{i}(\omega) g_{i}^{\prime}(u(\omega))\right.$,

$$
\left.\lambda(\omega) \geq 0, \lambda_{i}(\omega)=0 \text { if } i \notin I(\omega) \text {, a.e. } \omega \in \Omega\right\} .
$$

Let $u^{*} \in N_{K}(u)$, and $\lambda \in L_{\ell}^{s^{\prime}}(\Omega)$ be associated with $u^{*}$ by the above formula. Then

$$
|\lambda(\omega)| \leq a\left|u^{*}(\omega)\right| \text { a.e. on } \Omega,
$$

for some $a>0$ not depending on $u^{*}$, and

$$
T_{K}(u) \cap\left(u^{*}\right)^{\perp}=\left\{v \in T_{K}(u) ; \lambda_{i}(\omega) g_{i}^{\prime}(u(\omega)) v(\omega)=0, \text { a.e. } \omega \in \Omega\right\} .
$$

INRIA 
Proof.The formula for $T_{K}$ is proved in e.g. [22]. By lemma 4.2, we have that $u^{*} \in N_{K}(u)$ if and only if $u^{*} \in L_{\ell}^{s^{\prime}}(\Omega)$ and $u^{*}(\omega) \in N_{\underline{K}(\omega)}$, a.e. $\omega \in \Omega$. By (4.37), we have a.e. on $\Omega$ (e.g. [22])

$$
N_{\underline{K}(\omega)}=\left\{\sum_{i=1}^{q} \lambda_{i}(\omega) g_{i}^{\prime}(u(\omega)) ; \lambda(\omega) \geq 0, \lambda_{i}(\omega)=0 \text { if } i \notin I(\omega)\right\} .
$$

As $g$ is convex, $g\left(x_{0}\right)<0$ and $g_{i}(u(\omega))=0$ whenever $i \in I(u, \omega)$, we have

$$
g_{i}^{\prime}(u)\left(x_{0}-u(\omega)\right)<-a \text { if } i \in I(u, \omega) .
$$

It follows that

$$
\left\langle u^{*}(\omega),\left(x_{0}-u(\omega)\right)\right\rangle=\sum_{i=1}^{q} \lambda_{i}(\omega)\left\langle g_{i}^{\prime}(u(\omega)),\left(x_{0}-u(\omega)\right)\right\rangle \leq-a \sum_{i=1}^{q} \lambda_{i}(\omega) .
$$

Therefore,

$$
\sum_{i=1}^{q} \lambda_{i}(\omega) \leq a^{-1}\left|u^{*}(\omega)\right|\left|x_{0}-u(\omega)\right|
$$

This proves that $\lambda \in L_{q}^{s}(\Omega)$ whenever $u^{*} \in L_{\ell}^{s}(\Omega)$, as well as (4.40). Relation (4.41) is a consequence of (4.36iii). The conclusion follows.

We consider the Lagrangian function and the set of Lagrange multipliers

$$
\begin{aligned}
& \mathcal{L}(u, \lambda, \tau):= F(u, \tau)+\int_{\Omega}\langle\lambda(\omega), g(u(\omega))\rangle d \omega, \\
& \Lambda_{\tau}(u):=\left\{\lambda \in L_{\ell}^{s^{\prime}}(\Omega) ; F_{u}^{\prime}(u, \tau)+\sum_{i=1}^{q} \lambda_{i}(\omega) g_{i}^{\prime}(u(\omega))=0,\right. \\
&\left.\quad \lambda(\omega) \geq 0, \lambda_{i}(\omega)=0 \text { if } i \notin I(\omega), \text { a.e. } \omega \in \Omega\right\} .
\end{aligned}
$$

The second variation of the Lagrangian w.r.t. $u$ is defined as

$$
\mathcal{L}_{u^{2}}^{\prime \prime}(u, \lambda, \tau)(v, v):=F_{u^{2}}^{\prime \prime}(u, \tau)(v, v)+\int_{\Omega}\left\langle\lambda(\omega), g^{\prime \prime}(u(\omega))(v(\omega), v(\omega))\right\rangle d \omega .
$$

Note that whenever $u \in K \subset L_{\ell}^{\infty}(\Omega)$, then $F_{u}^{\prime}(u, \tau) \in H_{\ell}^{2}(\Omega)$ by assumption $\mathbf{A}(\mathrm{ii})$. By (2.2), $F_{u}^{\prime}(u, \tau) \in C_{\ell}(\bar{\Omega})$ if $n \leq 3$, and $F_{u}^{\prime}(u, \tau) \in L_{\ell}^{r}(\Omega), 1 / r=1 / 2-2 / n$, otherwise. If $\lambda$ is a Lagrange multiplier associated with $u$, by (4.40), $\lambda \in C_{q}(\bar{\Omega})$ if $n \leq 3$, and $\lambda \in L_{\ell}^{r}(\Omega)$ otherwise. As $s=2$ if $n \leq 3$, and $s>n / 2$ otherwise, the above integral is well defined.

We introduce the second-order necessary optimality condition as

$$
\forall v \in C(u), \quad \sup _{\lambda \in \Lambda_{\tau}(u)} \mathcal{L}_{u^{2}}^{\prime \prime}(u, \lambda, \tau)(v, v) \geq 0 .
$$

Under the assumptions of lemma 4.3, the set $\Lambda_{\tau}(u)$ is closed and bounded in $L_{q}^{s}(\Omega)$. In that case the supremum in (4.44) is attained. Note that the critical set $C(u)$ is here the set of directions satisfying the r.h.s. of (4.41), for some $\lambda \in \Lambda_{\tau}(u)$.

$\mathrm{RR} \mathrm{n}^{\circ} 3014$ 
Theorem 4.1 Let $g$ satisfy the assumptions of lemma 4.3. If $\bar{u}$ is a local solution of $S\left(P_{\tau}^{\prime}\right)$, and $K$ is of the form (4.38), then $u$ satisfies the second-order necessary condition (4.44).

Proof.Step 1. Denote by dist ${ }_{s}$ the distance in $L_{\ell}^{s}(\Omega)$. We check that the following metric regularity hypothesis holds:

$$
\forall \varepsilon_{1}>0 ; \exists M_{1}>0 ; \operatorname{dist}_{s}(u, K) \leq M_{1}\left\|g(u)_{+}\right\|_{s} \text { whenever }\|u\|_{\infty} \leq 1 / \varepsilon_{1} .
$$

Remind that $x_{0}$ is such that $a:=-\max _{i} g_{i}\left(x_{0}\right)>0$. As $g$ is convex, it follows that when $t=t(\omega) \in(0,1)$,

$$
g\left((1-t) u+t x_{0}\right) \leq(1-t) g(u)+t g\left(x_{0}\right) \leq(1-t) g(u)_{+}-t a .
$$

Set

$$
\bar{t}(\omega):=\frac{g(u(\omega))_{+}}{a+g(u(\omega))_{+}} .
$$

Then $g\left(u(\omega)+\bar{t}(\omega)\left(x_{0}-u(\omega)\right) \leq 0\right.$ by the above estimate, i.e. $u+\bar{t}\left(x_{0}-u\right) \in K$. Therefore

$$
\operatorname{dist}_{s}(u, K) \leq\left\|\bar{t}\left(x_{0}-u\right)\right\|_{s} \leq a^{-1}\left\|x_{0}-u\right\|_{\infty}\left\|g(u)_{+}\right\|_{s} .
$$

Hence (4.45) holds with $M_{1}:=a^{-1}\left(\left\|x_{0}\right\|_{\infty}+1 / \varepsilon_{1}\right)$.

Step 2: By proposition 4.1, the set

$$
\bar{C}(u):=\left\{v \in C(u) ; \exists \varepsilon>0 ; g_{i}(u)+\varepsilon g_{i}^{\prime}(u) v \leq 0, i \in I(u, \cdot) \text { a.e. on } \Omega\right\}
$$

is dense in $C(u)$. Furthermore, the set $\bar{C}(u) \cap L_{\ell}^{\infty}(\Omega)$ is dense in $\bar{C}(u)$. Indeed, let $v \in \bar{C}(u)$. Define $v_{\varepsilon}$ by

$$
v_{\varepsilon}(\omega)=v(\omega) \text { if }\|v(\omega)\| \leq \varepsilon^{-1} ; \quad 0 \text { otherwise. }
$$

Then by lemma $4.2, v_{\varepsilon} \in \bar{C}(u) \cap L_{\ell}^{\infty}(\Omega)$ and $v_{\varepsilon} \rightarrow v$ in $L_{\ell}^{s}(\Omega)$ as $\varepsilon \downarrow 0$.

The supremum in (4.44) being a continuous function of $v$ in $L_{\ell}^{s}(\Omega)$ (as $\Lambda_{\tau}(u)$ is closed and bounded), it suffices to prove that this supremum is nonnegative when $v$ is restricted to $\bar{C}(u) \cap L_{\ell}^{\infty}(\Omega)$.

Step 3: Fix $v \in \bar{C}(u) \cap L_{\ell}^{\infty}(\Omega)$. Let $z \in L_{\ell}^{\infty}(\Omega)$ be such that

$$
g_{i}^{\prime}(u) z+g_{i}^{\prime \prime}(u)(v, v) \leq 0 \text { whenever } i \in I(u, \cdot) \text {, a.e. on } \Omega \text {. }
$$

We check that $u_{t}:=u+t v+\frac{t^{2}}{2} z$ satisfies

$$
\operatorname{dist}_{s}\left(u_{t}, K\right)=o\left(t^{2}\right) .
$$

As $u, v$ and $z$ belong to $L_{\ell}^{\infty}(\Omega)$, and $g$ is smooth, we have

$$
g\left(u_{k}\right)=g(u)+t_{k} g^{\prime}(u) v_{k}+\frac{t_{k}^{2}}{2} g^{\prime \prime}(u)\left(v_{k}, v_{k}\right)+o\left(t_{k}^{2}\right) \text { in } L_{q}^{\infty}(\Omega) .
$$

INRIA 
Therefore, whenever $i \in I(u, \omega)$, we have $g_{i}\left(u_{t}(\omega)\right) \leq o_{1}\left(t^{2}\right)$, with $o_{1}$ (identified with its extension by 0 if $i \notin I(u, \omega))$ such that $t^{-2} o_{1}\left(t^{2}\right) \rightarrow 0$ in $L_{q}^{\infty}(\Omega)$.

On the other hand, let $\varepsilon>0$ be such that (4.47) holds. If $i \notin I(u, \omega)$, then by (4.47), for $t \leq \varepsilon / 2$, expanding $g(u(\omega))$ up to the second order, we have

$$
g_{i}\left(u_{t}(\omega)\right) \leq-\frac{\varepsilon}{2} g_{i}\left(u_{t}(\omega)\right)+t^{2} M,
$$

for some $M>0$ not depending on $\omega$. Therefore, $t^{-2} g_{i}\left(u_{t}\right)_{+} \leq M$, and $t^{-2} g_{i}\left(u_{t}\right)_{+} \rightarrow 0$ a.e. on $\Omega$. By Lebesgue's theorem $t^{-2} g_{i}\left(u_{t}\right)_{+} \rightarrow 0$ in $L_{q}^{s}(\Omega)$. This means that $g\left(u_{t}\right)+o_{2}\left(t^{2}\right) \in K$, where $t^{-2}\left\|o_{2}\left(t^{2}\right)\right\|_{s} \rightarrow 0$. By (4.45), it follows that there exists a feasible path of the form $u_{t}+o_{3}\left(t^{2}\right)$, with $t^{-2}\left\|o_{3}\left(t^{2}\right)\right\|_{s} \rightarrow 0$.

Step 4. Let $v$ and $z$ be as in step 3. As $v$ is a critical direction, it follows that

$$
F(u) \leq F\left(u_{t}+o_{3}(t)\right) \leq F(u)+\frac{t^{2}}{2}\left(F^{\prime}(u) z+F^{\prime \prime}(u)(v, v)\right)+o\left(t^{2}\right),
$$

hence $F^{\prime}(u) z+F^{\prime \prime}(u)(v, v) \geq 0$. It follows from the above discussion that the following problem parameterized by the function space $Z$ :

$$
\operatorname{Min}_{z \in Z} F^{\prime}(u) z+F^{\prime \prime}(u)(v, v) \text { s.t. } g_{i}^{\prime}(u) z+g_{i}^{\prime \prime}(u)(v, v) \leq 0, \quad i \in I(u, \cdot) .
$$

has, for the choice $Z=L_{\ell}^{\infty}(\Omega)$, a nonnegative value.

Let us consider now the case when $Z=L_{\ell}^{s}(\Omega)$. Denote by $F\left(S P_{0}\right)$ the feasible set of $\left(S P_{0}\right)$. We will show that $F\left(S P_{0}\right) \cap L_{\ell}^{\infty}(\Omega)$ is dense in $F\left(S P_{0}\right)$. As the cost function of $\left.S P_{0}\right)$ is continuous, this will imply that the infimum of $\left(S P_{0}\right)$ is still nonnegative when $Z=L_{\ell}^{s}(\Omega)$.

So, let $z \in F\left(S P_{0}\right)$. Consider the following truncation of $z$ :

$$
z_{\varepsilon}^{0}:=\max \left(-\varepsilon^{-1}, \min \left(z, \varepsilon^{-1}\right)\right) .
$$

Set

$$
\alpha_{\varepsilon}(\omega):=\left[g^{\prime}(u) z_{\varepsilon}^{0}+g_{i}^{\prime \prime}(u)(v, v)\right]_{+}(\omega) \text { if } i \in I(u, \omega), \quad 0 \text { otherwise. }
$$

Then

$$
\alpha_{\varepsilon}(\omega) \leq g_{i}^{\prime}(u)(z)_{+}+g_{i}^{\prime \prime}(u)(v, v)_{+}, \quad i \in I(u, \omega) .
$$

As $g_{i}^{\prime \prime}(u)(v, v) \in L_{q}^{\infty}(\Omega)$ and $z \in F\left(S P_{0}\right), g_{i}^{\prime}(u)(z)_{+} \in L_{q}^{\infty}(\Omega)$. It follows that as $\varepsilon \downarrow 0, \alpha_{\varepsilon}$ is bounded in $L_{q}^{\infty}(\Omega)$. Also $z_{\varepsilon}^{0} \rightarrow z$ in $L_{q}^{s}(\Omega)$, whence $\alpha_{\varepsilon}(\omega) \rightarrow 0$ a.e. on $\Omega$. By Lebesgue's theorem, $\alpha_{\varepsilon}(\omega) \rightarrow 0$ in $L_{q}^{s}(\Omega)$. Therefore the function

$$
z_{\varepsilon}^{1}:=z_{\varepsilon}^{0}+\frac{\alpha_{\varepsilon}}{a}\left(x_{0}-u\right)
$$

converges to $z$ in $L_{\ell}^{s}(\Omega)$, and by (4.42), belongs to $F\left(S P_{0}\right) \cap L_{\ell}^{\infty}(\Omega)$.

Step 5: we use duality theory in order to show that the value of $\left(S P_{0}\right)$ (with $Z=L_{\ell}^{s}(\Omega)$ ) is the supremum in (4.44). Indeed, this supremum is the dual (in the sense of convex duality)

$\mathrm{RR} \mathrm{n}^{\circ} 3014$ 
of $\left(S P_{0}\right)$. A sufficient condition for the primal and dual values to be equal is that the perturbed problem parameterized by $w \in L_{q}^{s}(\Omega)$

$$
\operatorname{Min}_{z} F^{\prime}(u) z+F^{\prime \prime}(u)(v, v) \text { s.t. } g_{i}^{\prime}(u) z+g_{i}^{\prime \prime}(u)(v, v) \leq w \text { whenever } i \in I(u, \cdot),
$$

is feasible whenever $\|w\|_{s}$ is small enough [22]. Set

$$
z_{0}:=a^{-1}\left(w-g_{i}^{\prime \prime}(u)(v, v)\right)_{+}\left(x_{0}-u\right) .
$$

In view of (4.42), $z_{0} \in L_{\ell}^{s}(\Omega)$, and is a feasible point of the above problem. The result follows.

Consider now the weak quadratic growth condition (note that we restrict $v$ to a small neighborhood of $v$ in $L_{\ell}^{\infty}(\Omega)$, and not $L_{\ell}^{s}(\Omega)$ as in $(2.17)$ )

$$
\begin{aligned}
& \exists \varepsilon>0 ; \quad \exists \alpha>0 ; \quad \text { if } v \in K, \quad\|v-u\|_{\infty} \leq \varepsilon ; \\
& F(v) \geq F(u)+\alpha\|v-u\|_{2}^{2}+o\left(\|v-u\|_{2}^{2}\right),
\end{aligned}
$$

the weak second order sufficient optimality condition

$$
\exists \alpha>0 ; \quad \forall v \in C(u), \quad \sup _{\lambda \in \Lambda_{\tau}(u)} \mathcal{L}_{u^{2}}^{\prime \prime}(u, \lambda, \tau)(v, v) \geq \alpha\|v\|_{2}^{2},
$$

as well as the punctual relation

$$
\forall v \in C(u) \backslash\{0\}, \quad \sup _{\lambda \in \Lambda_{\tau}(u)} \mathcal{L}_{u^{2}}^{\prime \prime}(u, \lambda, \tau)(v, v)>0 .
$$

Theorem 4.2 Let $K$ be as in lemma 4.3. Then the weak quadratic growth condition (4.52), the weak second-order sufficient condition (4.53) and (4.54) are equivalent.

Proof.By theorem 4.1, (4.52) implies (4.53). That (4.53) implies (4.54) is immediate. It remains to prove that (4.54) implies (2.17). If this is not true, then there exists a sequence $u_{k} \rightarrow u$ in $L_{\ell}^{2}(\Omega)$ such that

$$
F\left(u_{k}\right)<F(u)+\frac{1}{k}\left\|u_{k}-u\right\|_{2}^{2}
$$

and we may assume that $u_{k}=t_{k} v_{k}$, with $t_{k} \in \mathbb{R}_{+*}, t_{k} \downarrow 0,\left\|v_{k}\right\|_{2}=1$, and $v_{k} \stackrel{w}{\rightarrow} \bar{v}$ in $L_{\ell}^{2}(\Omega)$. On the other hand, let $\beta>0$. As $K$ is bounded in $L_{\ell}^{\infty}(\Omega)$ and $g$ is smooth, we have whenever $\left\|u_{k}-u\right\|_{\infty} \leq \varepsilon$, for $\varepsilon>0$ small enough:

$$
g\left(u_{k}\right) \geq g(u)+t_{k} g^{\prime}(u) v_{k}+\frac{t_{k}^{2}}{2} g^{\prime \prime}(u)\left(v_{k}, v_{k}\right)-\beta t_{k}^{2}\left|v_{k}\right|^{2} \quad \text { a.e. on } \Omega .
$$

Pick $\lambda \in \Lambda_{\tau}(u)$. From (4.56) and $\lambda \in L_{q}^{\infty}(\Omega)$, it follows that

$$
\mathcal{L}\left(u_{k}, \lambda, \tau\right) \geq \mathcal{L}(u, \lambda, \tau)+\frac{t_{k}^{2}}{2} \mathcal{L}_{u^{2}}^{\prime \prime}(u, \lambda, \tau)\left(v_{k}, v_{k}\right)-\beta t_{k}^{2}\|\lambda\|_{\infty}
$$

INRIA 
Using $\mathcal{L}(u, \lambda, \tau)=F(u), \mathcal{L}\left(u_{k}, \lambda, \tau\right) \leq F\left(u_{k}\right)$ and (4.55), we obtain $F^{\prime}(u) \bar{v}=0$ and $\lim \sup _{k} \mathcal{L}_{u^{2}}^{\prime \prime}(u, \lambda, \tau)\left(v_{k}, v_{k}\right) \leq \beta\|\lambda\|_{\infty}$, for all positive $\beta$. By (4.43) and assumption $\mathbf{A}(\mathrm{ii})$, $\mathcal{L}_{u^{2}}^{\prime \prime}(u, \lambda, \tau)$ is a Legendre form on $L_{\ell}^{2}(\Omega)$. Therefore $\bar{v} \neq 0$, while $\mathcal{L}_{u^{2}}^{\prime \prime}(u, \lambda, \tau)(\bar{v}, \bar{v}) \leq 0$. This contradicts (4.54).

We now pass to the perturbation analysis. The reader has noticed that the technique for obtaining upper (resp. lower) estimates of the value function is similar to the one for proving second order necessary (esp. sufficient) conditions. However, the second order sufficient condition above applies only in a small neighborhood of $L_{\ell}^{\infty}(\Omega)$. Therefore, we are not able to obtain lower estimates of the perturbed value function assuming (4.53). For that reason we state the second order upper estimate only. The subproblem is now

$$
\operatorname{Min}_{v} \max _{\lambda \in \Lambda_{\tau}(u)} \mathcal{L}^{\prime \prime}(u, \lambda, \tau)((v, \eta),(v, \eta)) \text {, s.t. }(2.4) \text { and } v \in C(\bar{u}),
$$

where by $\mathcal{L}^{\prime \prime}$ we mean the second derivative with respect to $(u, \tau)$.

Assumption A'.

Assumption $\mathbf{A}$ holds and, in addition, if $\left\{u_{k}\right\}$ is bounded in $L^{s}(\Omega)$ and $u_{k} \rightarrow u \in L^{2}(\Omega)$, then $F^{\prime}\left(u_{k}\right) \stackrel{w}{\rightarrow} F^{\prime}(u)$ in $H_{\ell}^{2}(\Omega)$.

This assumption is satisfied for the example discussed at the beginning of the section; in fact we have strong convergence of $F^{\prime}\left(u_{k}\right)$ in $H_{\ell}^{2}(\Omega)$ in that case. The strenghtening of $\mathbf{A}^{\prime}$ is needed for obtaining the first order lower estimate of the value of the perturbed problem, i.e. the inequality corresponding to (3.31).

Theorem 4.3 Assume that the coercivity hypothesis (2.5) holds, and that assumption A' holds. Let $\tau_{k}=\bar{\tau}+t_{k} \eta$. Let $u_{k} \in S\left(P_{\tau_{k}}^{\prime}\right)$. Then there exists $\bar{u} \in S\left(P_{\bar{\tau}}^{\prime}\right)$ such that, extracting a subsequence if necessary, $\bar{u}$ is the weak limit in $L_{\ell}^{s}(\Omega)$ and strong limit in $L_{\ell}^{2}(\Omega)$ of a subsequence of $\left\{u_{k}\right\}$, and is solution of

$$
\operatorname{Min}_{u}\left\langle\bar{\tau}-y_{u}, \eta\right\rangle_{2} ; u \in S\left(P_{\bar{\tau}}^{\prime}\right) .
$$

In addition, for the considered subsequence, we have

$$
\operatorname{val}\left(t_{k}\right) \leq \operatorname{val}\left(P_{\bar{\tau}}^{\prime}\right)+t_{k}\left\langle\bar{\tau}-y_{\bar{u}}, \eta\right\rangle_{2}+\frac{t_{k}^{2}}{2} \operatorname{val}\left(S P^{\prime}\right)+o\left(t_{k}^{2}\right) .
$$

Acknowlegdments. The author thanks Professors K. Malanowski and Tröltzsch for their useful remarks.

\section{References}

[1] S. Agmon, A. Douglis, L. Nirenberg, Estimates near the boundary for solutions of elliptic partial differential equations satisfying general boundary conditions I. Communications on Pure and Applied Mathematics 12 (1959), pp. 623-727.

$\mathrm{RR} \mathrm{n}^{\circ} 3014$ 
[2] R.A. Adams, Sobolev spaces. Academic Press, New York, 1975.

[3] H. BRÉzis, Problèmes unilatéraux. J. Mathématiques pures et appliquées 51 (1972), pp. $1-168$.

[4] J. F. Bonnans, The tangent optimization problem. Volume edited by A.V. Fiacco, Marcel Dekker, to appear.

[5] J. F. Bonnans and E. Casas, An extension of Pontryagin's principle for stateconstrained optimal control of semilinear elliptic equations and variational inequalities. SIAM J. Control Optimization 33 (1995), pp. 274-298.

[6] J.F. Bonnans and R. Cominetti, Perturbed optimization in Banach spaces I: a general theory based on a weak directional constraint qualification. SIAM J. Control Optimization 34(1996), pp. 1151-1171.

[7] J.F. Bonnans And A. Shapiro, Optimization problems with perturbations: A guided tour. Rapport de Recherche INRIA 2872, 1996.

[8] J.F. Bonnans And A. Shapiro, Perturbation analysis of optimization problems, in preparation.

[9] E. Casas, F. Tröltzsch And A. Unger, Second order sufficient optimality conditions for a nonlinear elliptic boundary control problem, Zeitschrift für Analysis und ihre Anwendungen 15 (1996), pp. 687-707.

[10] E. Casas and F. TRÖltzsch, Second order necessary optimality conditions for some state-constrained control problems of semilinear elliptic equations, Preprint 96-9 (1996), Fakultät für Mathematik, Technische Universität Chemnitz-Zwickau, D-09107 Chemnitz, Germany.

[11] R. Cominetti, Metric regularity, tangent sets and second order optimality conditions, Applied Mathematics and Optimization, 21 (1990), pp. 265-287.

[12] D. Gilbarg and N.S. Trudinger, Elliptic partial differential equations of second order. Springer Verlag, Berlin, 1983.

[13] A. Haraux, How to differentiate the projection on a convex set in Hilbert space. Some applications to variational inequalities, J. Mathematical Society of Japan (29) (1977), pp. $615-631$.

[14] A. D. Ioffe And V. M. Tinomirov, Theory of Extremal Problems, North-Holland Publishing Company, Amsterdam, 1979.

[15] J. L. Lions, Contrôle optimal de systèmes gouvernés par des équations aux dérivées partielles. Dunod, Paris, 1968. 
[16] K. Malanowski, Second order conditions and constraint qualifications in stability and sensitivity analysis of solutions to optimization problems in Hilbert spaces, Applied Mathematics and Optimization, 25 (1992), pp. 51-79.

[17] K. MalanowsKi, Two-norm approach in stability and sensitivity analysis of optimization and optimal control problems, Advances in Mathematical Sciences and Applications, 2 (1993), pp. 397-443.

[18] K. Malanowski, Stability and sensitivity of solutions to nonlinear optimal control problems. Applied Mathematics and Optimization, 32 (1995), pp. 111-141.

[19] H. Maurer And J. Zowe, First and second-order necessary and sufficient optimality conditions for infinite-dimensional programming problems, Mathematical Programming, 16 (1979), pp. 98-110.

[20] F. Mignot, Contrôle dans les inéquations variationnelles elliptiques, J. Functional Analysis 22 (1976), 25-39.

[21] S.M. Robinson, Strongly regular generalized equations, Mathematics of Operations Research 5 (1980), pp. 43-62.

[22] R.T. Rockafellar, Conjugate Duality and Optimization, Regional Conference Series in Applied Mathematics, SIAM, Philadelphia, 1974.

[23] J. Sokolowski, Sensitivity analysis of control constrained optimal control problems for distributed parameter systems. SIAM J. Control Optimization 25 (1987), pp. 1542-1556.

$\mathrm{RR} \mathrm{n}^{\circ} 3014$ 
Unité de recherche INRIA Lorraine, Technopôle de Nancy-Brabois, Campus scientifique, 615 rue du Jardin Botanique, BP 101, 54600 VILLERS LÈS NANCY

Unité de recherche INRIA Rennes, Irisa, Campus universitaire de Beaulieu, 35042 RENNES Cedex

Unité de recherche INRIA Rhône-Alpes, 655, avenue de l'Europe, 38330 MONTBONNOT ST MARTIN

Unité de recherche INRIA Rocquencourt, Domaine de Voluceau, Rocquencourt, BP 105, 78153 LE CHESNAY Cedex

Unité de recherche INRIA Sophia-Antipolis, 2004 route des Lucioles, BP 93, 06902 SOPHIA-ANTIPOLIS Cedex

Éditeur
INRIA, Domaine de Voluceau, Rocquencourt, BP 105, 78153 LE CHESNAY Cedex (France)

ISSN 0249-6399 